\title{
Predicting Falls in People with Multiple Sclerosis: Fall History Is as Accurate as More Complex Measures
}

\author{
Michelle H. Cameron, ${ }^{1}$ Emily Thielman, ${ }^{2}$ Rajarshi Mazumder, ${ }^{3}$ and Dennis Bourdette \\ ${ }^{1}$ Department of Neurology, Oregon Health \& Science University and Portland VA Medical Center, Portland, OR 97219, USA \\ ${ }^{2}$ National Center for Rehabilitative Auditory Research, Portland VA Medical Center, Portland, OR 97219, USA \\ ${ }^{3}$ Oregon Health \& Science University, Portland, OR 97219, USA
}

Correspondence should be addressed to Michelle H. Cameron; cameromi@ohsu.edu

Received 9 July 2013; Accepted 28 August 2013

Academic Editor: Francesco Patti

Copyright (C) 2013 Michelle H. Cameron et al. This is an open access article distributed under the Creative Commons Attribution License, which permits unrestricted use, distribution, and reproduction in any medium, provided the original work is properly cited.

Background. Many people with MS fall, but the best method for identifying those at increased fall risk is not known. Objective. To compare how accurately fall history, questionnaires, and physical tests predict future falls and injurious falls in people with MS. Methods. 52 people with MS were asked if they had fallen in the past 2 months and the past year. Subjects were also assessed with the Activities-specific Balance Confidence, Falls Efficacy Scale-International, and Multiple Sclerosis Walking Scale-12 questionnaires, the Expanded Disability Status Scale, Timed 25-Foot Walk, and computerized dynamic posturography and recorded their falls daily for the following 6 months with calendars. The ability of baseline assessments to predict future falls was compared using receiver operator curves and logistic regression. Results. All tests individually provided similar fall prediction (area under the curve (AUC) $0.60-0.75$ ). A fall in the past year was the best predictor of falls (AUC 0.75 , sensitivity 0.89 , specificity 0.56 ) or injurious falls (AUC 0.69 , sensitivity 0.96 , specificity 0.41 ) in the following 6 months. Conclusion. Simply asking people with MS if they have fallen in the past year predicts future falls and injurious falls as well as more complex, expensive, or time-consuming approaches.

\section{Introduction}

Multiple sclerosis (MS) impairs cognition, muscle strength, muscle tone, sensation, coordination, and gait, all of which are associated with an increased risk for falls [1,2]. A number of studies demonstrate that people with MS fall frequently [37] and suffer from various sequelae of falls, including injury $[5,8,9]$ and death [10], fear of falling, and reduced participation in occupational and leisure activities [11, 12]. To appropriately initiate fall prevention interventions, one must be able to identify those people with MS at increased risk for falls. Although various physical tests and questionnaires correlate with fall risk in MS [13-16], the ideal measure, which is quick, easy, and has high predictive accuracy, has not been identified.

The simplest, quickest approach for predicting future falls is to ask about past falls, but the strength of the relationship between past and future falls, and as well as predictive performance in comparison to other more complex measures in MS, is not known. In this study we compared how well future falls could be predicted by past falls, questionnaires, clinical measures readily performed by a physician, and computerized dynamic posturography (CDP), the gold-standard measure of human standing balance and postural control.

The objective of this study was to identify, in subjects with MS, which of the following had the highest average sensitivity for predicting a fall or an injurious fall in the subsequent 6 months: a history of falls within the past year; responses to the Activities-specific Balance Confidence (ABC), Falls Efficacy Scale-International (FES-I) and Multiple Sclerosis Walking Scale-12 (MSWS-12) questionnaires; Expanded Disability Status Scale (EDSS) and Timed 25 Foot Walk (T25-FW) scores; Automatic Postural Response (APR) latency from CDP testing.

\section{Materials and Methods}

2.1. Standard Protocol Approvals, Registrations, and Patient Consents. This was a prospective cohort study carried out at 
a Department of Veterans' Affairs and an academic medical center in the Northwest USA. The Institutional Review Board at both centers approved the protocol, and written informed consent was obtained from all patients participating in the study. The subjects were 52 people with MS recruited from the outpatient MS specialty clinics of these centers and surrounding community neurology clinics in 2010 to 2011. Potential subjects were recruited using flyers posted at the clinics, by providing information at patient education programs and support groups, and by referral from clinic healthcare providers. The clinics serve a total of approximately 1400 people with MS annually. From this population, 112 potential subjects expressed interest in participating in the study and, of these, 58 met inclusion criteria and consented to participate. Fifty two subjects completed all measures analyzed and were included in this analysis (Figure 1).

2.2. Inclusion and Exclusion Criteria. Inclusion criteria were age from 18 to 50 years, clinically and MRI-confirmed diagnosis of MS (McDonald criteria 2005 [17]) of any subtype, mild-to-moderate MS-associated disability (EDSS score $\leq 6.0$ [18]), willing and intellectually able to understand and sign an informed consent and adhere to protocol requirements, able to complete a written daily record of falls for 6 months, community dwelling, and no clinically significant MS relapse within 30 days prior to baseline testing. Exclusion criteria were a self-reported condition other than MS known to affect balance or gait, unable to follow directions in English, unhealed fractures or other conditions conveying risk of injury during balance testing; blindness, or, unable to walk more than 100 meters.

\subsection{Variables}

2.3.1. Falls. Falls and injurious falls in the 6 months following the baseline assessment were assessed prospectively by subjects documenting their falls each day for 6 months on monthly fall calendars and returning these calendars at the end of each month. The calendar stated, "Please write in the number of falls you have each day. A fall is any unexpected event that results in you ending up on the ground, floor, or any lower surface." In addition, subjects were asked to document each month if they suffered any injuries as a result of any fall. Subjects were contacted by phone during the first week of the fall count to reminder them to count their falls and to answer any calendar-related questions. Subjects returned the completed fall calendar at the end of each month and if the calendar was not received within 1 week after the end of the month, subjects were contacted by phone to ask for the calendar to be sent in.

The history of falls over the past 2 and 12 months was assessed at baseline with a questionnaire designed specifically for this study that included the following questions: How many times have you fallen in the past 2 months? How many times have you fallen in the past year? Options for responses were $0,1,2,3,4,5-10$, and more than 10 times.

2.3.2. Subjectively Assessed Balance Confidence, Fear of Falling, and Walking. Balance confidence and fear of falling were

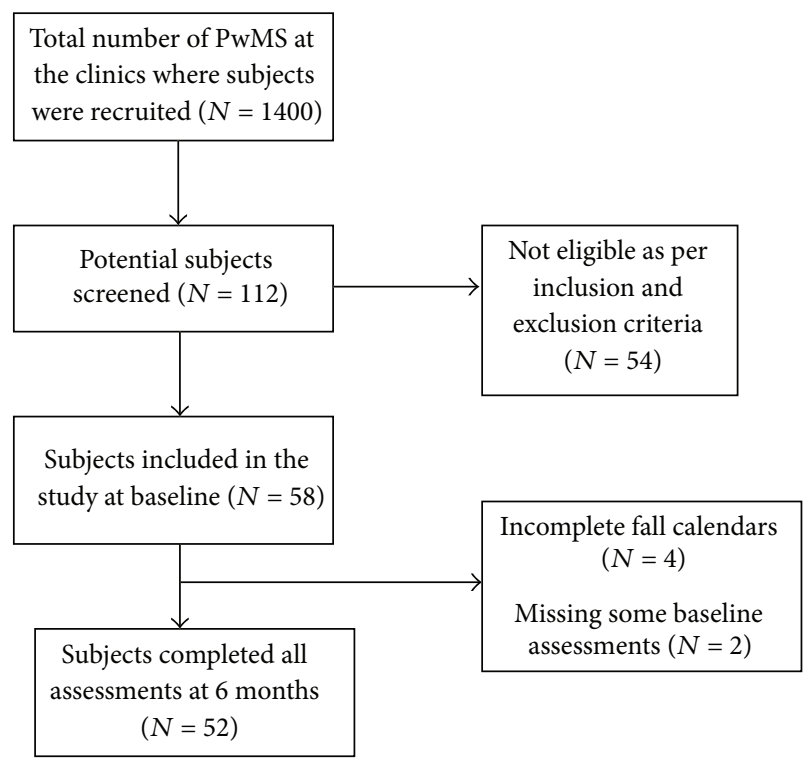

FIgURE 1: Participant flow diagram.

assessed at baseline with the ABC and the FES-I questionnaires, respectively $[19,20]$. Scores on these scales are associated with imbalance and fall risk in people with MS $[13,16,21]$. The ABC questionnaire asks subjects to rate their confidence in terms of whether they expect to lose balance or become unsteady when performing 16 mobility-related tasks. The FES-I questionnaire asks subjects to rate how concerned they are about falling when performing 16 activities of daily living. The MSWS-12 questionnaire was used to assess the self-reported impact of MS on walking [22]. The MSWS-12 questionnaire asks subjects to rate how much MS has limited 12 different aspects of their walking in the previous 2 weeks.

2.3.3. Clinically Assessed Walking. The EDSS [18] and T25FW [23] assessed overall MS-associated impairment and walking. The EDSS is an MS-specific measure scored based on a clinical neurological examination and the subject's walking capacity. EDSS scores are correlated with fall risk in MS $[4,21,24]$. The T25-FW is a component of the MS-specific clinical measure of disease-associated impairment, the Multiple Sclerosis Functional Composite (MSFC) [23]. For the T25-FW the subject is timed walking 25 feet as quickly as possible but safely. The relationship between T25-FW performance and fall risk has not been reported.

2.3.4. Computerized Balance Assessment. CDP objectively and precisely assessed standing balance performance. CDP uses commercially available specialized equipment to examine balance control. The subject stands on a force platform with a visual surround. During testing, the platform and/or the visual surround move under computer control and the platform measures the timing and force of the subject's postural responses. The delay between the platform moving backwards and the subject responding by exerting plantar flexion force on the platform is known as the automatic postural response (APR) latency. The APR latency is the most 
precisely measured and predominant balance abnormality in people with MS [25-29]. The relationship between APR latency and future fall risk in MS has not been reported. The composite APR latency from small, medium, and large backward translations was used for analysis in this study.

2.4. Statistical Methods. The goal of the analysis was to identify which of the baseline questionnaire, clinical, and computerized measures had the highest sensitivity for predicting the occurrence of any falls or any injurious falls in the following 6 months.

The probability of any falls or any injurious falls occurring in the 6 months following baseline assessment was modeled using logistic regression, with each predictor, including demographic characteristics and test performance, considered separately. Falls data from the calendars were categorized as zero and one or more falls or injurious falls during the entire 6-month follow-up period. Questionnaires were scored according to their scoring algorithms. The area under the receiver operating characteristic (ROC) curve (AUC) was computed from each logistic regression result. The ROC curve plots the true positive rate (predicted falls that actually occurred) against the false positive rate (predicted falls that did not actually occur). The AUC is the area under the ROC curve and provides a summary measure of the accuracy of the potential predictive variables. Thus, in this study, the AUC provides a measure of the average sensitivity for a specific baseline variable to predict a fall or an injurious fall within the following 6 months. The AUC ranges in value from 0.5 (chance) to 1.0 (perfect accuracy). Significance of the AUC measures was determined by repeated permutation testing.

Multivariate prediction models that combined two or more test components (e.g., APR from posturography and FES questionnaire score) were also considered. Logistic regression was performed using all analysis variables, and a stepwise selection process was employed to find the model that best predicts falls and injurious falls within the following 6 months. The selection process narrowed down the analysis variables to those that best contributed to the prediction model. Sensitivity, specificity, and positive and negative predictive values were computed for the variable with the highest AUC.

\section{Results}

3.1. Participants. Two-thirds of the participants were female. At baseline, their average age was about 40 years (mean 39.8 , range: 22 to 50 ) and the majority (94\%) had relapsingremitting MS (RRMS). The mean time since MS diagnosis was 6.3 years, the mean time since disease onset was 10.4 years, and the mean EDSS score was 2.8 (Table 1).

3.2. Falls and Test Performance. Thirty-seven (71\%) subjects fell at least once in the 6 months following baseline assessment, with a range from 1 to 21 falls in this period. Twentythree subjects (44\%) sustained at least one injury from a fall during this 6-month period. The subjects who were injured by a fall sustained bruises, cuts and grazes, sprains, and strains, and one subject had a collapsed lung, ruptured spleen, and
TABLE 1: Demographics and sample characteristics $(n=52)$.

\begin{tabular}{lc}
\hline Variable & Value \\
\hline Age: mean (SD, range) & $39.8(8.4,22-50)$ \\
Gender N (\%) & $35(67)$ \\
$\quad$ Female & $17(33)$ \\
$\quad$ Male & \\
MS subtype & 49 \\
$\quad$ RRMS & 3 \\
$\quad$ SPMS & 0 \\
$\quad$ PPMS & 0 \\
$\quad$ PRMS & $2.8(1.5,3.0,0-6)$ \\
EDSS: mean (SD, median, range) & $6.3(5.6,0-22)$ \\
Disease duration from diagnosis in years: & \\
mean (SD, range) & $10.4(8.3,0-31)$ \\
Disease duration from onset in years ${ }^{*}:$ mean & \\
(SD, range) &
\end{tabular}

${ }^{*}$ For one subject (included in analysis) date of disease onset was unknown.

TABLE 2: Types of injuries reported by the 23 subjects who were injured by a fall in the 6 months evaluated.

\begin{tabular}{ll}
\hline Type of injury & Number reported \\
\hline Bruise & 32 \\
Cut or graze & 25 \\
Sprain or strain & 3 \\
Pain only & 4 \\
& 3 (1 subject had a collapsed lung, spleen \\
rupture, and blood loss as the result of a fall. \\
1 subject lost a fingernail as the result of a \\
fall. 1 subject reported swelling associated \\
Other & and bruises, cuts or grazes, sprain or strain, \\
&
\end{tabular}

${ }^{*}$ The number of injuries was greater than the number of subjects injured by a fall as subjects could be injured by a fall more than once in the period and subjects could report more than one injury as the result of single fall.

blood loss as the result of a fall. Many subjects reported having pain associated with their injuries and four subjects reported only pain as the injury associated with a fall (Table 2 ).

Half of the subjects reported falling at least once in the 2 months prior to baseline. Of those who reported having fallen in the past 2 months, about half fell once and the rest fell 2 or more times. Three-quarters of the subjects reported falling at least once in the year prior to baseline, 7 subjects reported falling once in the past year, and 32 subjects reported multiple falls, including 4 who fell more than 10 times in the past year (Figure 2).

The mean ABC score for the sample was 78.8 (SD 20.9), the mean FES-1 score was 25.8 (SD 9.6), the mean MSWS12 score was 26.1 (SD 14.8), the mean 25 -foot walking speed was 5.7 seconds (SD 3.8), and the mean APR latency was 135.7 milliseconds (SD 19.7) (Table 3).

\subsection{Main Results}

3.3.1. Prediction of Falls and Injurious Falls in the following 6 Months. The AUC computed from logistic regression, with 


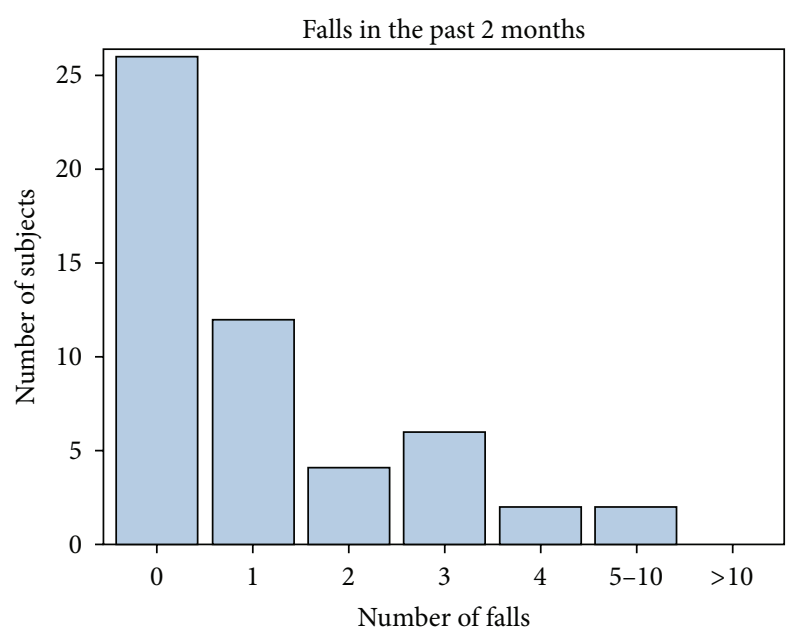

(a)

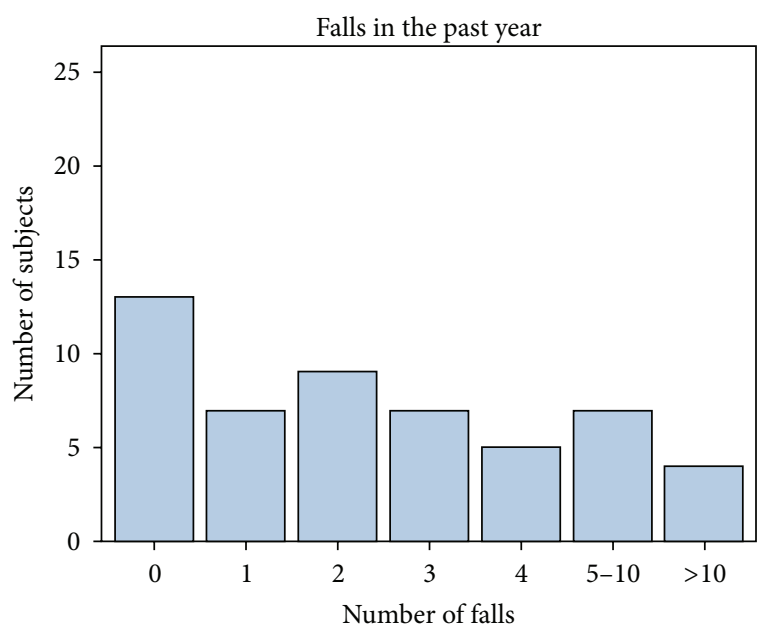

(b)

Figure 2: Number of falls sustained over the 2 months and 1 year prior to baseline.

each predictor, including demographic characteristics and test performance, considered separately or modeled together, demonstrated that falls in the past year had the highest sensitivity for predicting the occurrence of any falls or any injurious falls in the 6 months following baseline testing (AUC $=0.75$ for falls, 0.69 for injurious falls). Among the individual potential predictors, the EDSS score had the lowest AUC (0.60) for predicting any falls and the APR latency had the lowest AUC (0.52) for predicting any injurious falls (Table 3).

The AUC is a measure of the average predictive sensitivity, or accuracy, of a predictive variable. The AUC measures the average true positive rate (predicted falls that actually occurred) over all false positive rates (predicted falls that did not actually occur). In this study, the AUC provides a measure of the average sensitivity for a specific baseline variable to predict a fall or an injurious fall within the following 6 months.
3.3.2. Sensitivity, Specificity, and Predictive Value for Falls in the Past Year as Predictors of Falls and Injurious Falls in the following 6 Months. Reporting having one or more falls in the past year predicts that a subject will sustain one or more falls in the following 6 months with a sensitivity of 0.89 (95\% CI $0.73-0.96)$ and a specificity of 0.56 (95\% CI $0.31-0.79)$. The positive predictive value for this classification is $0.82(95 \%$ CI $0.66-0.92)$, and the negative predictive value is $0.69(95 \%$ CI 0.39-0.90). Reporting having one or more falls in the past year predicts that a subject will sustain one or more injurious falls in the following 6 months with a sensitivity of $0.96(95 \%$ CI $0.76-1.00)$ and a specificity of 0.41 (95\% CI $0.24-0.61)$. The positive predictive value for this classification is 0.56 (95\% CI $0.40-0.72)$, and the negative predictive value is 0.92 (95\% CI 0.62-1.00) (Table 4).

\section{Discussion}

This is the first prospective cohort study to demonstrate that, in people with MS, a history of a fall in the past year is a better predictor of falls and injurious falls in the following 6 months than questionnaires about balance or walking, clinical measures of MS-associated impairment or walking speed, or computerized dynamic posturography. Specifically, based on the data presented in this study, if a patient with MS reports falling in the past year they have an $82 \%$ probability of falling again in the following 6 months and a $56 \%$ probability of sustaining an injurious fall in the following 6 months. Equally importantly, if a patient with MS reports not falling in the past year, they have a $69 \%$ probability of not falling in the following 6 months and a $92 \%$ probability of not sustaining an injurious fall in the following 6 months. These test performance characteristics are well suited to clinical practice. The question, "Have you fallen in the past year?" is quick and easy to ask and accurately identifies those at high risk for falls and those not at high risk for injurious falls in the following 6 months.

The American Academy of Neurology Quality Standards Subcommittee practice parameter gives a level A recommendation for asking all patients in a neurology practice with fall risk factors about falls in the past year [1]. The fall risk factors identified in this practice parameter include disorders of gait and balance, use of assistive devices to ambulate, lower extremity weakness, or sensory loss. MS is not identified specifically as a fall risk factor but the parameter recommends that future research assesses the fall "risk for persons with specific neurologic conditions which may affect gait, mobility, or balance," and "identify screening tools that may be performed quickly and easily in the office or at the bedside." Prior falls have been found to be a strong predictor of future falls in older adults $[30,31]$ and in people with Parkinson's disease [32]. Our study demonstrates that prior falls are a strong predictor of future falls in MS. Our study also confirms the high frequency of falls in people with MS $[7,16,24]$. In our study $71 \%$ of subjects fell at least once in 6 months. Other studies prospectively assessing fall frequency in MS have reported $48 \%$ to $70 \%$ of subjects falling at least once in 3 to 12 months $[7,21,24]$. 
TABLE 3: Average sensitivity of baseline variables for predicting falls and injurious falls in the following 6 months.

\begin{tabular}{|c|c|c|c|}
\hline & Occurrence/score & $\begin{array}{l}\text { AUC: any falls in the } \\
\text { following } 6 \text { months }\end{array}$ & $\begin{array}{l}\text { AUC: any injurious falls in } \\
\text { the following } 6 \text { months }\end{array}$ \\
\hline A fall in the past year $n(\%)$ & $39(75 \%)$ & $\begin{array}{c}0.75 \\
P<0.001\end{array}$ & $\begin{array}{c}0.69 \\
P<0.001\end{array}$ \\
\hline A fall in the past 2 months $n(\%)$ & $26(50 \%)$ & $\begin{array}{c}0.71 \\
P=0.002\end{array}$ & $\begin{array}{c}0.64 \\
P=0.012\end{array}$ \\
\hline $\mathrm{ABC}$ total score & $78.8 \pm 20.9$ & $\begin{array}{c}0.69 \\
P=0.018\end{array}$ & $\begin{array}{c}0.66 \\
P=0.025\end{array}$ \\
\hline FES-I total score & $25.8 \pm 9.6$ & $\begin{array}{c}0.66 \\
P=0.029\end{array}$ & $\begin{array}{c}0.59 \\
P=0.14(\mathrm{NS})\end{array}$ \\
\hline MSWS-12 total score & $26.1 \pm 14.8$ & $\begin{array}{c}0.69 \\
P=0.013\end{array}$ & $\begin{array}{c}0.65 \\
P=0.031\end{array}$ \\
\hline $\begin{array}{l}\text { APR latency from posturography } \\
\text { (in milliseconds) }\end{array}$ & $135.7 \pm 19.7$ & $\begin{array}{c}0.62 \\
P=0.082\end{array}$ & $\begin{array}{c}0.52 \\
P=0.40(\mathrm{NS})\end{array}$ \\
\hline EDSS score & $2.8 \pm 1.5$ & $\begin{aligned} & 0.60 \\
P= & 0.14(\mathrm{NS})\end{aligned}$ & $\begin{array}{c}0.55 \\
P=0.30(\mathrm{NS})\end{array}$ \\
\hline T25FW time in seconds & $5.7 \pm 3.8$ & $\begin{array}{c}0.71 \\
P=0.009\end{array}$ & $\begin{array}{c}0.64 \\
P=0.043\end{array}$ \\
\hline
\end{tabular}

AUC: area under the curve; ABC: Activities-specific Balance Confidence; FES-I: Falls Efficacy Scale International; MSWS-12: Multiple Sclerosis Walking Scale12; APR: Automatic Postural Response; EDSS: Expanded Disability Status Scale; T25FW: Timed 25-Foot Walk.

TABLE 4: Association between falls in the past year and falls or injurious falls in the following 6 months.

\begin{tabular}{|c|c|c|c|c|}
\hline & \multicolumn{2}{|c|}{$\begin{array}{l}\text { Any falls in the } \\
\text { following } 6 \text { months }\end{array}$} & \multicolumn{2}{|c|}{$\begin{array}{l}\text { Any injurious falls in } \\
\text { the following } 6 \text { months }\end{array}$} \\
\hline & Yes & No & Yes & No \\
\hline \multicolumn{5}{|c|}{ Fell in the past year } \\
\hline Yes & 32 & 7 & 22 & 17 \\
\hline No & 4 & 9 & 1 & 12 \\
\hline
\end{tabular}

Limitations of this study include its moderate sample size, that it was carried out in a single location, that subjects were all aged 50 or under, that most (94\%) of the subjects had relapsing remitting MS, and that falls were self-reported on a calendar. However, the sample size was sufficient to demonstrate statistically and clinically significant relationships, and previous studies indicate that the incidence, timing and location of falls in people with MS are similar in different parts of the world [33]. Generalizability of the findings is limited because subjects were recruited from tertiary care MS centers in a single geographic region and they were recruited for a study of imbalance in MS, potentially selecting for people with greater imbalance and fall risk than in the general population of people with MS. Constraining the age of the cohort helped isolate the effects of MS on balance and fall risk at the cost of limiting generalizability to older people with MS. The high proportion of subjects with relapsing remitting MS was unexpected but may be related to restricting the age of the cohort to under 50 and the EDSS to 6.0 or less, biasing away from primary and secondary progressive MS both of which are associated with a later age of onset and higher level of disability than relapsing remitting MS. Although self-reporting of falls by subjects could have biased our findings, because there is no accurate alternative to selfreport, fall calendars are considered the gold standard for measuring falls prospectively [34]. Accelerometer-based fall detection methods are available, but these are limited by moderate specificity and poor sensitivity [35]. To assure data completeness, subjects in this study were contacted by phone to remind them to complete and return their fall calendars. Future studies would benefit from a larger sample size and a more diverse sample to enhance generalizability and to allow for further subgroup analyses.

One of the most important limitations of this study's findings is that predicting future falls based on previous falls requires the person to fall at least once. Ideally, one would predict and prevent a person's first fall. However, reassuringly, the data indicate that if a patient with MS reports not falling in the past year, although they may fall in the following 6 months, they are extremely unlikely to be injured by a fall in this time frame. In addition, only asking if a person has fallen in the past year will not identify the specific factors contributing to fall risk for the individual, which might be needed to select effective interventions to help prevent future falls. However, this simple approach quickly identifies those at increased fall risk for whom further discussion and testing may help direct selection of fall prevention interventions such as use of an assistive device, physical therapy assessment and treatment, medication changes, or multidimensional fall prevention education. In addition, just as MRI and relapse rate are used to select patients with active MS for trials of MS disease modifying therapy, this study suggests that a history of falls is an ideal tool for selecting those patients with MS at high risk for falls for participation in trials of fall prevention therapies.

\section{Conclusions}

In summary, simply asking people with MS if they have fallen in the past year predicts future falls and future injurious falls as well as more complex, expensive, or time-consuming 
approaches. In the context of a busy clinical practice this is feasible and certainly quicker and easier than other options. We recommend that clinicians ask all their MS patients whether they have fallen in the past year to easily identify those at increased risk for falling and for sustaining fall related injury.

\section{Glossary}

ABC: $\quad$ Activities-specific balance confidence

APR: $\quad$ Automatic postural response

AUC: Area under the curve

CDP: Computerized dynamic posturography

EDSS: $\quad$ Expanded disability status scale

FES-I: Falls efficacy scale international

MS: $\quad$ Multiple sclerosis

MSFC: Multiple sclerosis functional composite

MSWS-12: Multiple Sclerosis Walking Scale-12

ROC: $\quad$ Receiver Operating Curve

T25-FW: Timed 25-Foot Walk.

\section{Acknowledgments}

This study was supported by a Career Development Award to Michelle Cameron from the Department of Veterans Affairs, Rehabilitation Research \& Development Service the VA. Rajarshi Mazumder was supported by the Oregon Multidisciplinary Training Program in Health Services Research, Grant no. T32 HS017582 from the Agency for Health Research and Quality (AHRQ) and the 2012 Foundation of the Consortium of Multiple Sclerosis Centers. The funding granted to Michelle Cameron and to Rajarshi Mazumder is acknowledged.

\section{References}

[1] D. J. Thurman, J. A. Stevens, and J. K. Rao, "Practice parameter: assessing patients in a neurology practice for risk of falls (an evidence-based review): report of the Quality Standards Subcommittee of the American Academy of Neurology," Neurology, vol. 70, no. 6, pp. 473-479, 2008.

[2] J. J. Sosnoff, M. J. Socie, M. K. Boes et al., "Mobility, balance and falls in persons with multiple sclerosis," PLoS One, vol. 6, no. 11, Article ID e28021, 2011.

[3] D. Cattaneo, C. De Nuzzo, T. Fascia, M. Macalli, I. Pisoni, and R. Cardini, "Risks of falls in subjects with multiple sclerosis," Archives of Physical Medicine and Rehabilitation, vol. 83, no. 6, pp. 864-867, 2002.

[4] M. L. Finlayson, E. W. Peterson, and C. C. Cho, "Risk factors for falling among people aged 45 to 90 years with multiple sclerosis," Archives of Physical Medicine and Rehabilitation, vol. 87, no. 9, pp. 1274-1279, 2006.

[5] E. W. Peterson, C. C. Cho, L. von Koch, and M. L. Finlayson, "Injurious falls among middle aged and older adults with multiple sclerosis," Archives of Physical Medicine and Rehabilitation, vol. 89, no. 6, pp. 1031-1037, 2008.

[6] P. N. Matsuda, A. Shumway-Cook, A. M. Bamer, S. L. Johnson, D. Amtmann, and G. H. Kraft, "Falls in Multiple Sclerosis," $P M$ and $R$, vol. 3, no. 7, pp. 624-632, 2011.
[7] H. J. Gunn, S. Creanor, B. Haas, J. Marsden, and J. Freeman, "Risk-factors for falls in multiple sclerosis: an observational study," Multiple Sclerosis Journal, 2013.

[8] M. H. Cameron, A. J. Poel, J. K. Haselkorn, A. Linke, and D. Bourdette, "Falls requiring medical attention among veterans with multiple sclerosis: a cohort study," Journal of Rehabilitation Research and Development, vol. 48, no. 1, pp. 13-20, 2011.

[9] M. T. Bazelier, T. Van Staa, B. M. Uitdehaag et al., "The risk of fracture in patients with multiple sclerosis: the UK general practice research database," Journal of Bone and Mineral Research, vol. 26, no. 9, pp. 2271-2279, 2011.

[10] H. Brønnum-Hansen, T. Hansen, N. Koch-Henriksen, and E. Stenager, "Fatal accidents among Danes with multiple sclerosis," Multiple Sclerosis, vol. 12, no. 3, pp. 329-332, 2006.

[11] E. W. Peterson, C. C. Cho, and M. L. Finlayson, "Fear of falling and associated activity curtailment among middle aged and older adults with multiple sclerosis," Multiple Sclerosis, vol. 13, no. 9, pp. 1168-1175, 2007.

[12] P. N. Matsuda, A. Shumway-Cook, M. A. Ciol, C. H. Bombardier, and D. A. Kartin, "Understanding falls in multiple sclerosis: association of mobility status, concerns about falling, and accumulated impairments," Physical Therapy, vol. 92, no. 3, pp. 407-415, 2012.

[13] D. Cattaneo, A. Regola, and M. Meotti, "Validity of six balance disorders scales in persons with multiple sclerosis," Disability and Rehabilitation, vol. 28, no. 12, pp. 789-795, 2006.

[14] Y. Nilsagard, A. Carling, and A. Forsberg, "Activities-specific balance confidence in people with multiple sclerosis," Multiple Sclerosis International, vol. 2012, Article ID 613925, 8 pages, 2012.

[15] Y. Nilsagard, C. Lundholm, L.-G. Gunnarsson, and E. Dcnison, "Clinical relevance using timed walk tests and "timed up and go" testing in persons with multiple sclerosis," Physiotherapy Research International, vol. 12, no. 2, pp. 105-114, 2007.

[16] R. van Vliet, P. Hoang, S. Lord, S. Gandevia, and K. Delbaere, "Falls efficacy scale-international: a cross-sectional validation in people with multiple sclerosis," Archives of Physical Medicine and Rehabilitation, vol. 94, pp. 883-889, 2013.

[17] C. H. Polman, S. C. Reingold, G. Edan et al., "Diagnostic criteria for multiple sclerosis: 2005 revisions to the 'McDonald Criteria", Annals of Neurology, vol. 58, no. 6, pp. 840-846, 2005.

[18] J. F. Kurtzke, "Rating neurologic impairment in multiple sclerosis: an expanded disability status scale (EDSS)," Neurology, vol. 33, no. 11, pp. 1444-1452, 1983.

[19] L. E. Powell and A. M. Myers, "The Activities-specific Balance Confidence (ABC) scale," Journals of Gerontology A, vol. 50, no. 1, pp. M28-M34, 1995.

[20] L. Yardley, N. Beyer, K. Hauer, G. Kempen, C. Piot-Ziegler, and C. Todd, "Development and initial validation of the Falls Efficacy Scale-International (FES-I)," Age and Ageing, vol. 34, no. 6, pp. 614-619, 2005.

[21] Y. Nilsagård, C. Lundholm, E. Denison, and L.-G. Gunnarsson, "Predicting accidental falls in people with multiple sclerosisa longitudinal study," Clinical Rehabilitation, vol. 23, no. 3, pp. 259-269, 2009.

[22] J. C. Hobart, A. Riazi, D. L. Lamping, R. Fitzpatrick, and A. J. Thompson, "Measuring the impact of MS on walking ability: the 12-item MS Walking Scale (MSWS-12)," Neurology, vol. 60, no. 1, pp. 31-36, 2003.

[23] G. R. Cutter, M. L. Baier, R. A. Rudick et al., "Development of a multiple sclerosis functional composite as a clinical trial outcome measure," Brain, vol. 122, no. 5, pp. 871-882, 1999. 
[24] S. L. Kasser, J. V. Jacobs, J. T. Foley, B. J. Cardinal, and G. F. Maddalozzo, "A prospective evaluation of balance, gait, and strength to predict falling in women with multiple sclerosis," Archives of Physical Medicine and Rehabilitation, vol. 92, no. 11, pp. 1840-1846, 2011.

[25] M. H. Cameron, F. B. Horak, R. R. Herndon, and D. Bourdette, "Imbalance in multiple sclerosis: a result of slowed spinal somatosensory conduction," Somatosensory and Motor Research, vol. 25, no. 2, pp. 113-122, 2008.

[26] M. H. Cameron and S. Lord, "Postural control in multiple sclerosis: implications for fall prevention," Current Neurology and Neuroscience Reports, vol. 10, no. 5, pp. 407-412, 2010.

[27] D. Frzovic, M. E. Morris, and L. Vowels, "Clinical tests of standing balance: performance of persons with multiple sclerosis," Archives of Physical Medicine and Rehabilitation, vol. 81, no. 2, pp. 215-221, 2000.

[28] C. L. Martin, B. A. Phillips, T. J. Kilpatrick et al., "Gait and balance impairment in early multiple sclerosis in the absence of clinical disability," Multiple Sclerosis, vol. 12, no. 5, pp. 620-628, 2006.

[29] F. Soyuer, M. Mirza, and Ü. Erkorkmaz, "Balance performance in three forms of multiple sclerosis," Neurological Research, vol. 28, no. 5, pp. 555-562, 2006.

[30] M. E. Tinetti, M. Speechley, and S. F. Ginter, "Risk factors for falls among elderly persons living in the community," The New England Journal of Medicine, vol. 319, no. 26, pp. 1701-1707, 1988.

[31] A. J. Campbell, M. J. Borrie, and G. F. Spears, "Risk factors for falls in a community-based prospective study of people of 70 years and older," Journals of Gerontology, vol. 44, no. 4, pp. M112-M117, 1989.

[32] N. E. Allen, A. K. Schwarzel, and C. G. Canning, "Recurrent falls in Parkinson's disease: a systematic review," Parkinson's Disease, vol. 2013, Article ID 906274, 16 pages, 2013.

[33] M. H. Cameron and Y. Nilsagard, "Falls in people with MS: a transatlantic perspective," Neurology, vol. 80, Meeting Abstracts 1, P04.106, 2013.

[34] S. E. Lamb, E. C. Jørstad-Stein, K. Hauer, and C. Becker, “Development of a common outcome data set for fall injury prevention trials: the Prevention of Falls Network Europe consensus," Journal of the American Geriatrics Society, vol. 53, no. 9, pp. 1618-1622, 2005.

[35] F. Bagala, C. Becker, A. Cappello et al., "Evaluation of accelerometer-based fall detection algorithms on real-world falls," PLoS One, vol. 7, Article ID e37062, 2012. 


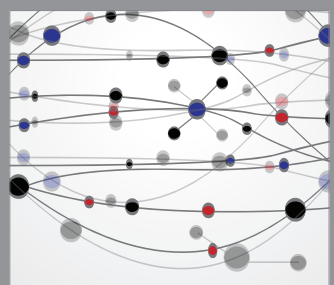

The Scientific World Journal
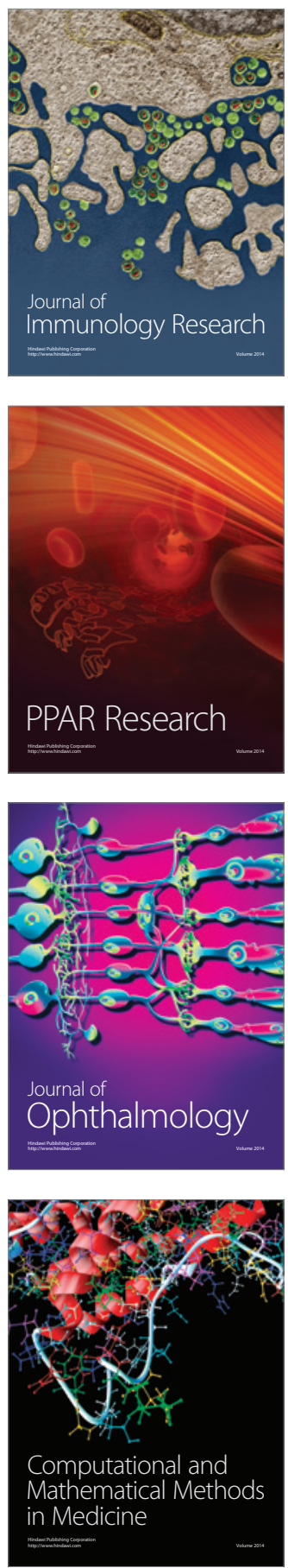

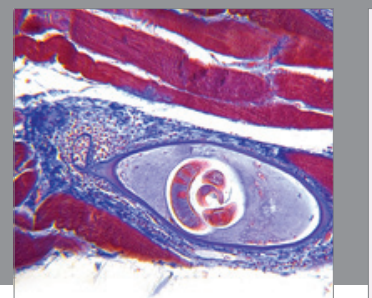

Gastroenterology

Research and Practice
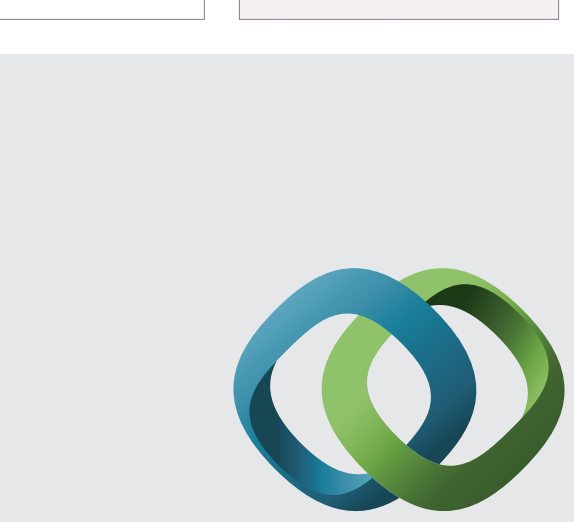

\section{Hindawi}

Submit your manuscripts at

http://www.hindawi.com
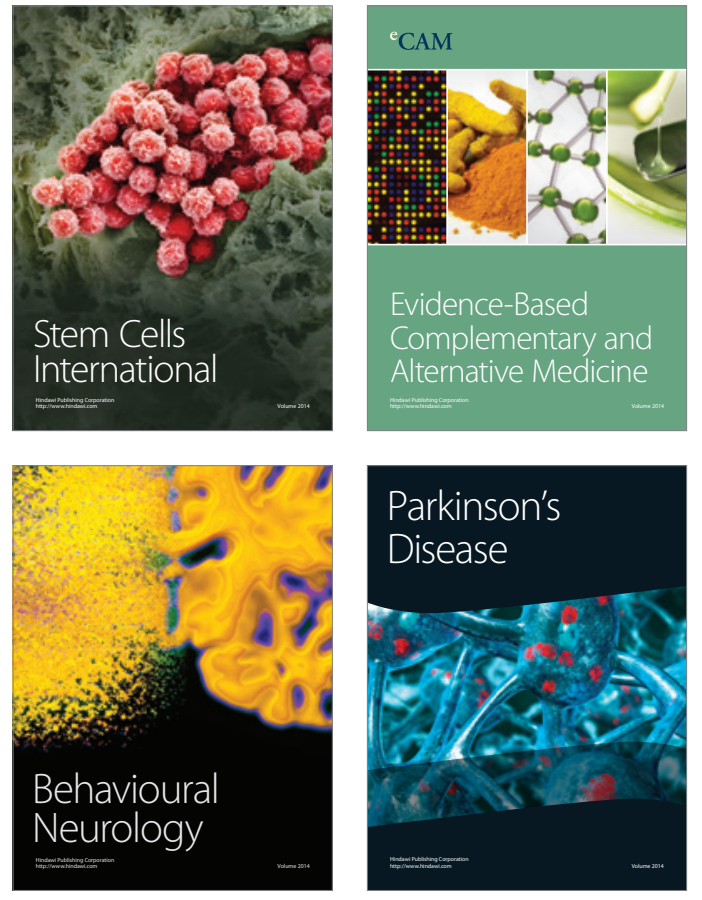
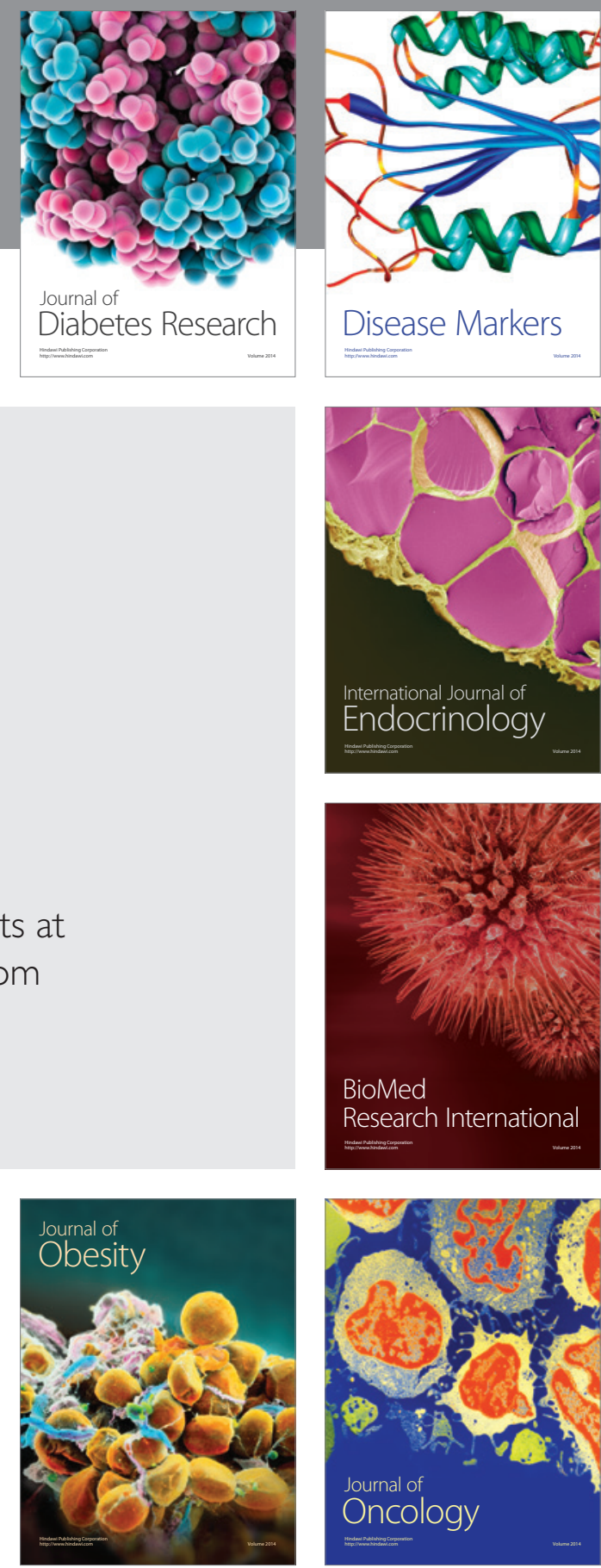

Disease Markers
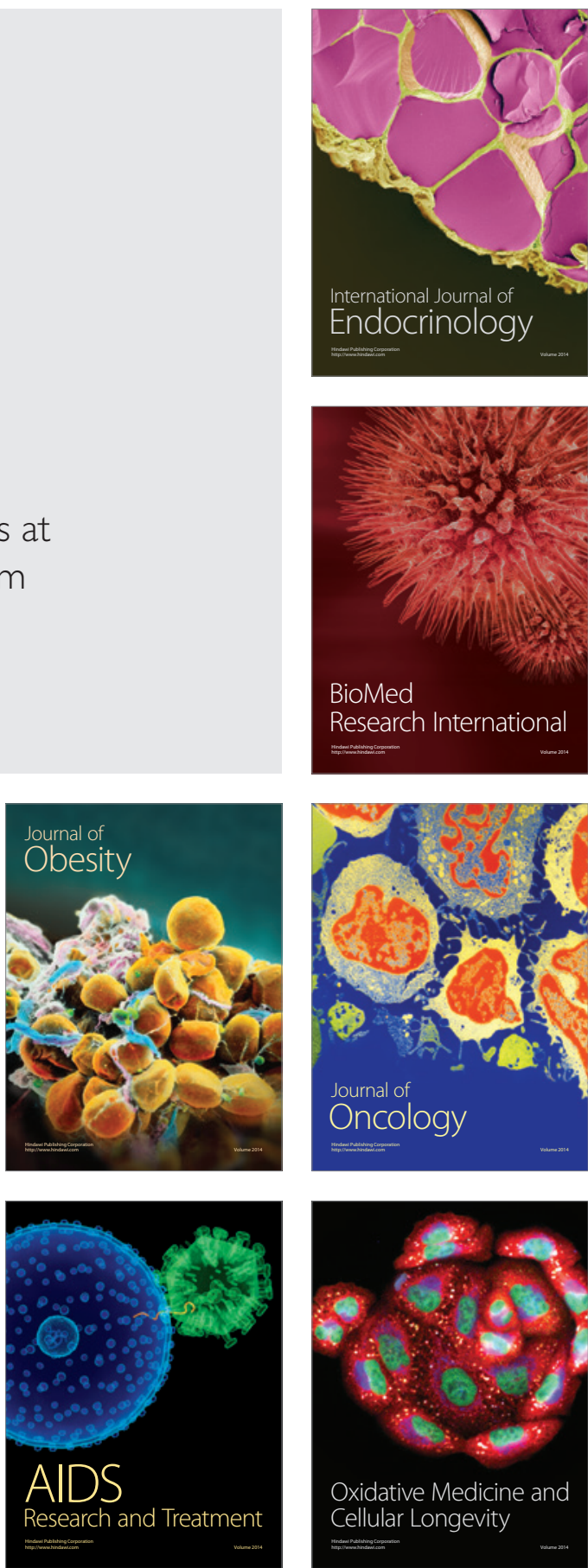